\title{
REPRESENTATION OF LINEAR FUNCTIONALS ON QUASI-CONTINUOUS FUNCTIONS
}

\author{
JAMES D. BAKER
}

\begin{abstract}
The question of representing the continuous linear functionals on the complex quasi-continuous functions in terms of a single integral of Stieltjes type is considered. An integral on three functions is defined, is shown to provide a unique representation of the functionals, and in special cases, is shown to reduce to standard Stieltjes integrals involving two functions.
\end{abstract}

1. Introduction. In 1934, Kaltenborn [1] extended the Riesz representation theorem to include functions having discontinuities of the first kind (i.e., the quasi-continuous functions). This representation had the form of a modified Stieltjes integral plus an infinite sum. Others, including [3], have shown that a solution could be obtained with the sum of two integrals; however, it has been of mathematical interest as to whether or not a single integral of Stieltjes type could be used.

Perhaps the most complete solution to date is contained in an unpublished result of R. E. Lane which states that the continuous, stationary linear operators on the quasi-continuous functions can be expressed as

$$
\Omega f(s)=(m) \int_{-\infty}^{+\infty} f(s-u(t)) d v(t),
$$

where the functions $u$ and $v$ depend on $\Omega$, and $(m)$ denotes the Stieltjes mean integral. The linear functionals are then obtained by considering $\Omega f(0)$. It has not been demonstrated, however, that all the linear functionals can be obtained from this restricted class of operators. Properties of the se operators can be found in [2]. Also, one of the deficiencies of the result in [3] is that the representation is not unique since different integrator func tions can be used for a given functional.

In this paper, the method of representation involves an integral on three functions. The integral is defined in $\$ 2$, which also contains the necessary

Presented to the Society, January 28, 1973; received by the editors October 6 , 1972 and, in revised form, December 13, 1973.

AMS (MOS) subject classifications (1970). Primary 28A25, 28A40.

Key words and phrases. Stieltjes integrals, quasi-continuous functions, linear functionals. 
existence conditions. The representation and uniqueness theorems follow in $\$ 3$.

2. Preliminaries. Let $B$ denote a complex Banach space; let $g$ and $b$ be functions with domain the closed interval $[a, b]$ and range in $B$; and let $f$ be a complex-valued function on $[a, b]$. By $\int_{a}^{b}(g, b) d f$, we mean the refinement limit of the sums

$$
\sum_{i=1}^{n}\left\{g\left(t_{i-1}\right)\left[f\left(\zeta_{i}\right)-f\left(t_{i-1}\right)\right]+b\left(t_{i}\right)\left[f\left(t_{i}\right)-f\left(\zeta_{i}\right)\right]\right\}
$$

where $\left\{a=t_{0}<t_{1}<\cdots<t_{n}=b\right\}$ is a partition of $[a, b], \zeta_{i} \in\left(t_{i-1}, t_{i}\right)$ for $i=1,2, \cdots, n$, and where the limit is taken in terms of the norm of $B$.

Let $Q C$ denote the Banach space of quasi-continuous complex-valued functions with domain the closed interval $[a, b]$ and with the supremum norm. Let $\lambda$ be the function with domain $[a, b]$ and range in $Q C$ such that for each $t \in[a, b], \lambda(t)$ is the real-valued function with domain $[a, b]$ such that

$$
\begin{aligned}
& \lambda(t)(x)=0 \text { for } a \leq x \leq t \text {, } \\
& =1 \text { for } t<x \leq b \text {. }
\end{aligned}
$$

Similarly, let $\rho$ be the function with domain $[a, b]$ and range in $Q C$ such that for each $t \in[a, b], \rho(t)$ is the real-valued function with domain $[a, b]$ such that

$$
\begin{aligned}
& \rho(t)(x)=0 \text { for } a \leq x<t \text {, } \\
& =1 \text { for } t \leq x \leq b \text {. }
\end{aligned}
$$

We observe that if $f$ is a complex-valued step function with domain $[a, b]$, there exists a partition $P=\left\{a=t_{0}<t_{1}<\cdots<t_{n}=b\right\}$ of $[a, b]$ such that if $\zeta_{i} \in\left(t_{i-1}, t_{i}\right)$ for $i=1,2, \cdots, n$, then

$$
\begin{aligned}
f & =\sum_{i=0}^{n} f\left(t_{i}\right)\left[\rho\left(t_{i}\right)-\lambda\left(t_{i}\right)\right]+\sum_{i=1}^{n} f\left(\zeta_{i}\right)\left[\lambda\left(t_{i-1}\right)-\rho\left(t_{i}\right)\right] \\
& =f(a) \rho(a)+\int_{a}^{b}(\lambda, \rho) d f .
\end{aligned}
$$

By approximating $f$ with a sequence of step-functions, the representation in (2.2) can be shown to hold for all $f \in Q C$.

'Theorem 2.1. Suppose $g$ and $b$ are complex-valued functions of bounded variation on $[a, b]$, and suppose $g-b$ vanishes except at a countable number 
of points in $[a, b]$. If $f \in Q C$, then $\int_{a}^{b}(g, b) d f$ exists. Moreover, if $\left\{f_{n}\right\}$ is a sequence of functions in $Q C$ which converge uniformly to $f$, then $\lim _{n \rightarrow \infty} \int_{a}^{b}(g, b) d f_{n}=\int_{a}^{b}(g, b) d f$.

Proof. The sums (2.1) can be written as

$$
\begin{aligned}
& \sum_{i=0}^{n}\left[g\left(t_{i-1}\right)-b\left(t_{i-1}\right)\right]\left[f\left(\zeta_{i}\right)-f\left(t_{i-1}\right)\right] \\
& \quad+f(b) b(b)-f(a) b(a)-\sum_{i=0}^{n} f\left(\zeta_{i}\right)\left[b\left(t_{i}\right)-b\left(t_{i-1}\right)\right] .
\end{aligned}
$$

Let $\left\{f_{m}\right\}$ denote a sequence of step functions in $Q C$ which converge to $f$ uniformly on $[a, b]$. Let $P=\left\{a=t_{0}<t_{1}<\cdots<t_{n}=b\right\}$ denote a partition of $[a, b]$, and let $\zeta_{i} \in\left(t_{i-1}, t_{i}\right)$ for $i=1,2, \cdots n$. We introduce the following notation:

$$
\begin{aligned}
S(P . m) & =\sum_{i=1}^{n}\left[g\left(t_{i-1}\right)-b\left(t_{i-1}\right)\right]\left[f_{m}\left(\zeta_{i}\right)-f_{m}\left(t_{i-1}\right)\right], \\
S(P) & =\sum_{i=1}^{n}\left[g\left(t_{i-1}\right)-b\left(t_{i-1}\right)\right]\left[f\left(\zeta_{i}\right)-f\left(t_{i-1}\right)\right], \\
S(m) & =\sum_{t \in[a, b)}[g(t)-b(t)]\left[f_{m}\left(t^{+}\right)-f_{m}(t)\right], \\
S & =\sum_{t \in[a, b)}[g(t)-b(t)]\left[f\left(t^{+}\right)-f(t)\right] .
\end{aligned}
$$

We have: $\lim _{P} S(P, m)=S(m)$ since $f_{m}$ is a step function; $\lim _{m} S(P, m)=$ $S(P)$ uniformly in $P$ since $g-b$ is of bounded variation and vanishes except at a countable number of points; and $\lim _{m} S(m)=S$. Using iterated limits, we have

$$
\lim _{P} S(P)=\lim _{P} \lim _{m} S(P, m)=\lim _{m} S(m)=S .
$$

Thus, the refinement limit of the first sum in equation (2.3) is $S$; and the limit of the second is $(I) \int_{a}^{b} f d b$. The second part of the the orem follows because $g$ and $b$ are of bounded variation and because of the assumption regarding $(g-b)$.

In the example that follows, we show that the hypotheses $g$ and $b$ are of bounded variation and $f \in Q C$ are not sufficient for $\int_{a}^{b}(g, b) d f$ to exist.

Example 2.1. For $t \in[0,1]$, let $f(t)=1 / m$ if $t=(1-1 / m), m=1,2, \cdots$, and let $f(t)=0$ otherwise; for $t \in[0,1]$, let $g(t)=0$ and $b(t)=1$. Let $P=$ $\left\{t_{0}<t_{1}<\cdots<t_{n}\right\}$ be a partition of $[0,1]$, and suppose $m_{0}$ is the smallest 
integer such that $\left(1-1 / m_{0}\right)>t_{n-1}$. It is possible to choose a refinement $P^{\prime}=\left\{t_{0}^{\prime}<t_{1}^{\prime}<\cdots<t_{n+p}^{\prime}\right\}$ of $P$ and a sequence $\left\{\zeta_{i}^{\prime}\right\}, i=1,2, \cdots, n+p$, such that

$$
\left|\sum_{i=0}^{n}\left[f\left(t_{i}\right)-f\left(\zeta_{i}\right)\right]-\sum_{i=1}^{n+p}\left[f\left(t_{i}^{\prime}\right)-f\left(\zeta_{i}^{\prime}\right)\right]\right|=\sum_{i=0}^{p-1} \frac{1}{\left(m_{0}+i\right)} .
$$

Theorem 2.2. If $g$ is a complex-valued function on $[a, b]$, then $\int_{a}^{b}(g, g) d f$ exists if and only if $(I) \int_{a}^{b} f d g$ exists, and in this case,

$$
\int_{a}^{b}(g, g) d f=f(b) g(b)-f(a) g(a)-(I) \int_{a}^{b} f d g
$$

Proof. For this case, (2.1) becomes

$$
\sum_{i=1}^{n}\left\{f\left(t_{i}\right) g\left(t_{i}\right)-f\left(t_{i-1}\right) g\left(t_{i-1}\right)-f\left(\zeta_{i}\right)\left[g\left(t_{i}\right)-g\left(t_{i-1}\right)\right]\right\} .
$$

This result relates $\int_{a}^{b}(g, g) d f$ to both the Young integral $(Y) \int_{a}^{b} g d f$ and the weighted integral $(1,-1,1) \int_{a}^{b} g d f[4]$.

3. Representation. The integral discussed above is used to characterize continuous linear functionals on $Q C$. In this case, the value of the functional is allowed to be complex.

Theorem 3.1. If $T$ is a continuous complex-valued linear functional on $Q C$, there are complex-valued functions $\alpha$ and $\beta$ on $[a, b]$ such that if $f \in Q C$, then

$$
T(f)=f(a) \beta(a)+\int_{a}^{b}(\alpha, \beta) d f
$$

Proof. Let $\alpha(t)=T(\lambda(t))$ and $\beta(t)=T(\rho(t))$. Let $M$ be a positive real number such that $|T(f)| \leq M\|f\|$ for each $f \in Q C$. For any partition of $P=$ $\left\{a=t_{0}<t_{1}<\cdots<t_{n}=b\right\}$ of $[a, b]$, there exists a sequence of complex numbers $\left\{\epsilon_{i}\right\}, i=0,1, \cdots, n$, with $\left|\epsilon_{i}\right|=1$ such that

$$
\sum_{i=0}^{n}\left|(\alpha-\beta)\left(t_{i}\right)\right|=\sum_{i=0}^{n} \epsilon_{i}(\alpha-\beta)\left(t_{i}\right)=\left|T\left(\sum_{i=0}^{n} \epsilon_{i}(\lambda-\rho)\left(t_{i}\right)\right)\right| \leq M .
$$

Thus $\alpha-\beta$ vanishes except at a countable number of points in $[a, b]$. Similarly, $\alpha$ and $\beta$ are shown to be of bounded variation on $[a, b]$. If $f \in Q C$, then $\int_{a}^{b}(\alpha, \beta) d f$ exists as a result of Theorem 2.1; and if $f_{s}$ is a step function in $Q C$, then from (2.2), 


$$
T\left(f_{s}\right)=\beta(a) f_{s}(a)+\int_{a}^{b}(\alpha, \beta) d f_{s} .
$$

The theorem follows from Theorem 2.1 and the continuity of $T$.

For each $x \in[a, b], \lambda(b)(x)=0$, so $a(b)=T(\lambda(b))=0$. If we anchor the function $a$ at $b$, this insures the following result.

Theore $\mathrm{m}$ 3.2. The representation in Theorem 3.1 is unique.

Proof. Suppose $\hat{\alpha}$ and $\hat{\beta}$ are of bounded variation, $\hat{\alpha}(b)=0$, and $(\hat{\alpha}-\hat{\beta})$ vanishes except at a countable number of points. Then $U(f)=$ $\hat{\beta}(a) f(a)+\int_{a}^{b}(\hat{\alpha}, \hat{\beta}) d f$ defines a continuous linear functional on $Q C$. In addition, suppose $T(f)=U(f)$ for each $f \in Q C$. Then

$$
f(a)[\beta(a)-\hat{\beta}(a)]+\int_{a}^{b}(\alpha-\hat{a}, \beta-\hat{\beta}) d f=0 .
$$

By choosing $f(x)=\rho(a)(x)$ for $x \in[a, b]$, we have $\beta(a)=\hat{\beta}(a)$. If $t \in[a, b)$, then

$$
\int_{a}^{b}[(\alpha-\hat{\alpha})(x),(\beta-\hat{\beta})(x)] d \lambda(t)(x)=\alpha(t)-\hat{\alpha}(t)=0 ;
$$

likewise, if $t \in(a, b]$, then

$$
\int_{a}^{b}[(\alpha-\hat{\alpha})(x),(\beta-\hat{\beta})(x)] d \rho(t)(x)=\beta(t)-\hat{\beta}(t)=0 .
$$

\section{REF ERENCES}

1. H. S. Kaltenborn, Linear functional operations on functions having discontinuities of the first kind, Bull. Amer. Math. Soc. 40 (1934), 702-708.

2. R. E. Lane, Linear operators on quasi-continuous functions, Trans. Amer. Math. Soc. 89 (1958), 378-394. MR 20 \#5416.

3. W. W. Schmaedeke and G. R. Sell, The Gronwall inequality for modified Stieltjes integrals, Proc. Amer. Math. Soc. 19 (1968), 1217-1222. MR 37 \#6422.

4. F. M. Wright and J. D. Baker, On integration-by-parts for weighted integrals, Proc. Amer. Math. Soc. 22 (1969), 42-52. MR $39 \# 7056$.

HONEYWELL CORPORATE RESEARCH CENTER, BLOOMINGTON, MINNESOTA 55420 